\title{
PAGES FROM THE COMPUTER FILES OF R. WILLIAM GOSPER
}

\author{
MOURAD E. H. ISMAIL, YU TAKEUCHI, AND RUIMING ZHANG
}

(Communicated by Charles Pugh)

\begin{abstract}
We give proofs of summation theorems and continued fraction evaluations conjectured by R. W. Gosper. We also give two new elementary proofs of a theorem of Gosper whose original proof uses matrix methods. One proof uses iteration of two term recurrence relation. The latter proof is also used to give elementary proofs of three other identities due to Gosper.
\end{abstract}

\section{INTRODUCTION}

The theory of hypergeometric and basic hypergeometric functions provides a canonical way of writing many combinatorial identities and, together with complex analytic techniques, have provided a systematic way of proving combinatorial identities. Recently, however, $R$. William Gosper, through very clever computer experimentation with the computer algebra package Macsyma, formulated many identities which do not fit the existing theory of hypergeometric functions and $q$ series as in [15, 3], for example. He developed new techniques to prove certain identities and formulated many conjectures that led to very interesting developments [4]-[6]. This paper is the second installment of a serious attempt to go through Gosper's computer files and correspondence. The first installment was [11]. The purpose of this series of papers is to explain where Gosper's formulas come from and to prove his conjectures.

An interesting identity of Gosper [8] is

$$
\begin{aligned}
& 1+\sum_{n=1}^{\infty}(\sqrt{x}-c)(\sqrt{\sqrt{x}}-c) \cdots\left(x^{2^{-n}}-c\right) \\
& \quad=\frac{1+x / c}{c+1}, \quad|1-c|<1, \quad x \notin(-\infty, 0) .
\end{aligned}
$$

For a proof see $[7, \S 5]$. Gosper used his matrix technique and his theory of path invariance and telescopy to prove (1.1). In [9] Gosper mentioned the identity,

Received by the editors August 16, 1991.

1991 Mathematics Subject Classification. Primary 33A10, 30B10; Secondary 33A40, 30B50.

Research partially supported by NSF grants DMS 8814026, DMS 8912423, and INT 8803099 and also by the Instituto Colombiano Para El Fomento De La Education Superior. 
due to Gene Salamin,

(1.2)

$$
\sum_{n=1}^{\infty}\left(n \pi+\arctan \frac{n \pi+\arctan \frac{n \pi+\arctan \ldots}{3}}{3}\right)^{-2}=(3 \tanh (3 \tanh 3 \ldots))^{-2} .
$$

Gosper [9] conjectured the continued fraction evaluations

$$
\begin{aligned}
c \sinh x & +\frac{1}{c \sinh (x+y)+\frac{1}{c \sinh (x+2 y)+\cdots}} \\
= & c \sinh x \frac{\sum_{n=0}^{\infty} \frac{\exp \{-n[(n-1) y+x]\}}{\prod_{k=1}^{n} c \sinh (k y) c \sinh [(k-1) y+x]}}{\sum_{n=0}^{\infty} \frac{\exp [-n(n y+x)]}{\prod_{k=1}^{n} c \sinh (k y) c \sinh (k y+x)}} \\
c-y-1 / y-\frac{1}{c z-y-1 / y-\frac{1}{c z^{2}-y-1 / y-\cdots}} & (c-1 / y) \frac{y^{2}}{\sum_{n=0}^{\infty} \prod_{k=1}^{n} \frac{\prod_{k=1}^{n}}{\left(1-z^{-k}\right)\left(1-c y z^{k-1}\right)}} \\
& =\frac{y^{2}}{\left(1-z^{-k}\right)\left(1-c y z^{k}\right)} \\
c z+\frac{1}{c z^{2}+\frac{1}{c z^{3}}+\cdots} & \sum_{n=0}^{\infty} \prod_{k=1}^{n} \frac{1}{\sum_{n=0}^{\infty} \prod_{k=1}^{n} \frac{1}{\left(z^{-k}-1\right)\left(1+c z^{k-1}\right)}}
\end{aligned}
$$

He also pointed out the following interesting consequence of (1.3) [9]:

$$
\lim _{y \rightarrow 0} \frac{\sum_{n=0}^{\infty} \frac{\exp \{-n[(n-1) y+x]\}}{\prod_{k=1}^{n} c \sinh (k y) c \sinh [(k-1) y+x]}}{\sum_{n=0}^{\infty} \frac{\exp [-n(n y+x)]}{\prod_{k=1}^{n} c \sinh (k y) c \sinh (k y+x)}}=\frac{1}{2}\left[1+\sqrt{1+4 /(c \sinh x)^{2}}\right],
$$

which follows from the continuity of the continued fraction in (1.3) as a function of $y$.

In $\S 2$ we give two proofs of (1.1) and a proof of the following generalization 
of (1.2):

$$
\begin{aligned}
& \sum_{n=1}^{\infty}\left(n \pi+\arctan \frac{\left.n \pi+\arctan \frac{n \pi+\arctan \ldots}{c}\right)^{-2}}{c}\right)^{-(c \tanh (c \tanh c \ldots))^{-2}+\frac{c-3}{6(c-1)}, \quad c>1 .}
\end{aligned}
$$

We also give a substantial generalization of (1.6) which enables us to replace the exponent 2 in the sum on the left side of (1.6) by any even exponent; see (2.6) and (2.7).

In the process of proving (1.1), in $\S 2$ we show that if $f$ is real analytic in a neighborhood of $x=1$ and satisfies the functional equation

$$
f\left(x^{2}\right)=1+(x-c) f(x)
$$

then

$$
f(x)=\frac{[1+x / c]}{c+1} .
$$

Both sides of (1.1) are obviously real analytic in $x$ in a neighborhood of $x=1$, and one can easily verify that they satisfy (1.7). This raises the question of whether (1.7) has other solutions under conditions weaker than real analyticity in a neighborhood of $x=1$. For remarks and a conjecture see the last part of $\S 2$. In [8] Gosper mentioned the identity

$$
\begin{gathered}
\sum_{n=0}^{\infty} \frac{c^{n}}{z^{(-1 / 2)^{n}}}\left(1-c \sqrt{z^{3}}\right)\left(1-\frac{c}{\sqrt{\sqrt{z^{3}}}}\right) \cdots\left(1-c z^{-3(-1 / 2)^{n+1}}\right) \\
=\frac{z^{-1}-c^{2} z^{2}}{1+c^{3}}, \quad|c(1-c)|<1 .
\end{gathered}
$$

It is interesting that ideas behind our proofs of (1.1) also establish (1.9). In particular, there is a simple principle of iterating identities involving rational functions that leads to formulas like (1.1) and (1.9). This together with a proof of (1.9) will also be discussed in $\S 2$.

In $\S 3$ we establish (1.3) and (1.4). We evaluate the continued fraction in (1.5) and seem to get an answer in a form different from the right-hand side in (1.5). We have not been able to transform our answer to Gosper's conjectured form. Our answer is $\left(1.5^{\prime}\right)$ at the end of $\S 3$. We point out that (1.3) is equivalent to a continued fraction of Al-Salam and Ismail [1]. We also show that the partial numerators and partial denominators on (1.3)-(1.5) can be expressed in closed form as single sums.

After we prepared a preliminary version of this paper Gosper [10] communicated to the first author the following trigonometric version of (1.1):

$$
\begin{gathered}
\sum_{n=1}^{\infty}\left\{\prod_{k=1}^{n}\left[-2 \cos \left(b+a /(-2)^{k}\right)\right]\right\} \sin \left[-3 b n-c+a /(-2)^{n}\right] \\
=\frac{\sin (2 b-a) \sin (c+b)}{\sin (3 b)}, \quad|\cos b|<\frac{1}{2}
\end{gathered}
$$


In $\S 4$ we will prove $(1.10)$ and the identities

$$
\begin{gathered}
\sum_{n=1}^{\infty}\left(-\frac{1}{2}\right)^{n} \frac{a+b z^{(-2)^{-n}}}{1+z^{(-2)^{-n}}}=\frac{a-b}{2}\left(\frac{2}{\ln z}+\frac{1+z}{1-z}\right)-\frac{a+b}{6}, \\
\sum_{n=1}^{\infty}(-1)^{n}\left(a z^{(-2)^{-n} / 3}+b z^{5(-2)^{-n} / 3}\right)\left(\sqrt[2^{n}]{z}-1 / \sqrt[2^{n}]{z}\right) \\
=\left(z^{-2 / 3}-1\right)\left[a+b\left(z^{-2 / 3}+1-z^{2 / 3}\right)\right] .
\end{gathered}
$$

The identities (1.11) and (1.12) are stated in [9], but the original version of (1.12) contained a minor typographical error. In $\S 4$ we will also prove the additional identity of Gosper

$$
\begin{aligned}
& \sum_{n=1}^{\infty} \frac{\sinh \left(2^{n} a-b n+c\right)}{\prod_{k=0}^{n-1}\left[2 \cosh \left(2^{k} a+b\right)\right]} \\
& \quad=\frac{\sinh (a+b)}{\sinh b}\left[\sinh (c-2 b)+\frac{1}{2} e^{2 b-c} \prod_{n=1}^{\infty} \frac{e^{b} \sinh \left(2^{n} a+b\right)}{\sinh \left(2^{n} a+2 b\right)}\right],
\end{aligned}
$$

$\operatorname{Re}(a)>0, \operatorname{Re}(b)>0,|\cosh (a+b)|<\frac{1}{2}$. Gosper noted that the infinite product in (1.13) converges very rapidly, like $\exp \left(-\alpha 2^{n}\right)$, but the series on the left-hand side converges at a much slower rate.

\section{Formulas (1.1), (1.6), AND (1.9)}

Our first proof of (1.1) is based on the functional equation (1.7), while the second proof uses the principle of iteration.

$A$ proof of (1.1). Let $f(x)$ denote the left-hand side of (1.1). Clearly

$$
f\left(x^{2}\right)=1+\sum_{n=1}^{\infty}(x-c)\left(x^{1 / 2}-c\right) \cdots\left(x^{2^{1-n}}-c\right)=1+(x-c) f(x),
$$

and (1.7) follows. Since $f(x)$ is analytic in the complex variable $x$ in a neighborhood of $x=1$, it is represented there by its Taylor series $f(x)=$ $\sum_{n=0}^{\infty} A_{n}(x-1)^{n}$. Now substitute the Taylor series of $f$ in the functional equation (1.7) to get

$$
\sum_{n=0}^{\infty} A_{n}(x-1)^{n}[2+(x-1)]^{n}=1+(1-c) \sum_{n=0}^{\infty} A_{n}(x-1)^{n}+\sum_{n=0}^{\infty} A_{n}(x-1)^{n+1},
$$

and upon equating coefficients of $(x-1)^{n}$ we obtain

$$
\begin{gathered}
A_{0}=1+(1-c) A_{0}, \\
\sum_{n \geq j \geq n / 2}\left(\begin{array}{c}
j \\
n-j
\end{array}\right) 2^{2 j-n} A_{j}=A_{n-1}+(1-c) A_{n}, \quad n>0 .
\end{gathered}
$$

So $A_{0}=1 / c, A_{1}=1 /[c(c+1)], A_{2}=A_{3}=0$, and the above recursion implies that $A_{n}=0, n \geq 3$. Thus $f(x)=1 / c+(x-1) /[c(c+1)]=(x+c) /[c(c+1)]$ and $(1.1)$ is proved.

$A$ second proof of (1.1). The idea is to observe that

$$
\frac{1+z / c}{1+c}=1+\frac{z-c^{2}}{c(c+1)}=1+(S z-c) \frac{S z+c}{c(c+1)}
$$


where $S$ is the square root operation: $S z=\sqrt{z}, z>0$. Since the term $(S z+c) /[c(c+1)]$ is the left-hand side with $z$ replaced by $\sqrt{z}$, we think of the operator $S$ as an iteration operator for the function $(z+c) /[c(c+1)]$. Now define $f_{n}(z)$ via

$$
f_{n}(z):=1+(S z-c)\left(1+\left(S^{2} z-c\right)\left(1+\cdots\left(1+S^{n} z-c\right) \cdots\right)\right) .
$$

A repeated application of the above process leads to

$$
\begin{aligned}
\frac{1+z / c}{c+1}-f_{n}(z) & =\left\{\prod_{k=1}^{n}\left(S^{k} z-c\right)\right\} \frac{\left(S^{n} z-c^{2}\right)}{c(c+1)} \\
& =\frac{1}{c(c+1)}\left\{\prod_{k=1}^{n+1}\left(S^{k} z-c\right)\right\}\left(S^{n+1} z+c\right) .
\end{aligned}
$$

Now let $n \rightarrow \infty$ and the extreme right-hand side in the above equality will tend to 0 very rapidly since $|1-c|<1$ and $S^{n} z \rightarrow 1$ as $n \rightarrow \infty$. This completes the proof of (1.1).

It is worth noting that our second proof gives the rate of convergence of $f_{n}(z)$ to the function $(1+z / c) /(c+1)$. The convergence is clearly exponential. Gosper [7] observed the fast convergence.

$A$ proof of (1.9). This proof uses functional equations. The term $n=0$ in the sum on the left-hand side of $(1.9)$ is $\left(1-c z^{3 / 2}\right) / z$, so we set

$$
f(z):=z \sum_{n=0}^{\infty} \frac{c^{n}}{z^{(-1 / 2)^{n}}}\left(1-c \sqrt{z^{3}}\right)\left(1-\frac{c}{\sqrt{\sqrt{z^{3}}}}\right) \cdots\left(1-c z^{-3(-1 / 2)^{n+1}}\right)
$$

Since $f(z)$ involves mostly $z^{3}$ and we will expand $f(z)$ in a neighborhood of $z=1$, we set $u=z^{3}$ and $g(u)=f(z)$.

From (2.1) it is clear that

$$
\begin{aligned}
f\left(z^{2}\right)=1-c z^{3}+z^{2}\left(1-c z^{3}\right) \sum_{n=1}^{\infty} \frac{c^{n}}{z^{2(-1 / 2)^{n}}}\left(1-\frac{c}{\sqrt{z^{3}}}\right) & \left(1-\frac{c}{\sqrt{\sqrt{z^{3}}}}\right) \\
& \cdots\left(1-c z^{3(-1 / 2)^{n}}\right) ;
\end{aligned}
$$

hence, upon replacing $n$ by $n+1$ in the above sum we find

that is,

$$
f\left(z^{2}\right)=1-c u+c u(1-c u) f(1 / z) \text {, }
$$

$$
g\left(u^{2}\right)=1-c u+c u(1-c u) g(1 / u) .
$$

If $g(u)=\sum_{n \geq 0} A_{n}(u-1)^{n}$, then the above functional equation gives $A_{0}=$ $(1-c) /\left(1-c+c^{2}\right)$. Furthermore,

$$
g\left(u^{2}\right)=\sum_{m=0}^{\infty}(u-1)^{m} \sum_{m \geq n \geq m / 2}\left(\begin{array}{c}
n \\
m-n
\end{array}\right) 2^{2 n-m} A_{n}
$$

and

$$
\begin{aligned}
g\left(\frac{1}{u}\right) & =\sum_{k=0}^{\infty} A_{k}(1-u)^{k} u^{-k}=A_{0}+\sum_{k=1}^{\infty}(-1)^{k} A_{k}(u-1)^{k}(1+u-1)^{-k} \\
& =A_{0}+\sum_{m=1}^{\infty}(1-u)^{m} \sum_{n=1}^{m} A_{n} \frac{(n)_{m-n}}{(m-n) !} .
\end{aligned}
$$


Now the substitution of the above expansions in (2.2) and equating coefficients of like powers of $u-1$ implies $A_{0}=(1-c) /\left(1-c+c^{2}\right)$ and $A_{1}=-c^{2} /\left(1+c^{3}\right)$. For $m \geq 2$ we compute the $A_{n}$ 's recursively from

$$
\begin{aligned}
(-1)^{m} & \sum_{m \geq n \geq m / 2}\left(\begin{array}{c}
n \\
m-n
\end{array}\right) 2^{2 n-m} A_{n} \\
= & c(1-c) \sum_{n=0}^{m} A_{n} \frac{(n)_{m-n}}{(m-n) !}+c(2 c-1) \sum_{n=0}^{m-1} A_{n} \frac{(n)_{m-n-1}}{(m-n-1) !} \\
& -c^{2} \sum_{n=0}^{m-2} A_{n} \frac{(n)_{m-n-2}}{(m-n-2) !},
\end{aligned}
$$

and conclude, by induction, that $A_{m}=0$ for $m \geq 2$. This completes the proof. $A$ proof of (1.9) by iteration. Start with the identity

$$
\frac{1-c^{2} z^{3}}{z\left(1+c^{3}\right)}=\left(1-c z^{3 / 2}\right)\left[\frac{1}{z}+\frac{c \sqrt{z}}{1+c^{3}}\left(1-\frac{c^{2}}{\sqrt{z^{3}}}\right)\right]
$$

then observe that the second term in the square bracket is the left-hand side with $z$ replaced by $1 / \sqrt{z}$. This is the idea of iteration. Define the iteration operator $S$, which acts only on the variable $z$, by $S z=1 / \sqrt{z}$. Then define $f_{m}(z)$ as

$$
f_{m}(z)=\sum_{n=0}^{m} \frac{c^{n}}{z^{(-1 / 2)^{n}}}\left(1-c \sqrt{z^{3}}\right)\left(1-\frac{c}{\sqrt{\sqrt{z^{3}}}}\right) \cdots\left(1-c z^{-3(-1 / 2)^{n+1}}\right) .
$$

It is then clear that

$$
\begin{aligned}
\frac{1-c^{2} z^{3}}{z\left(1+c^{3}\right)}-f_{m}(z)= & c^{m+1}\left(1-c \sqrt{z^{3}}\right)\left(1-\frac{c}{\sqrt{\sqrt{z^{3}}}}\right) \\
& \cdots\left(1-c z^{-3(-1 / 2)^{m+1}}\right) S^{m+1}\left(\frac{1-c^{2} z^{3}}{z\left(1+c^{3}\right)}\right),
\end{aligned}
$$

and thus the partial sums $f_{m}(z)$ converge to $\left(1-c^{2} z^{3}\right) /\left[z\left(1+c^{3}\right)\right]$. Thus the proof is complete.

Proof of (1.6). It is clear that the transcendental equation

$$
x=c \tanh x
$$

has a unique positive real root $L$, say, when $c>1$. The successive approximations

$$
x_{1}=c, \quad x_{k+1}=c \tanh x_{k}, \quad k=1,2, \ldots,
$$

converge to $L$. On the other hand the transcendental equation

$$
x=c \tan x, \quad c>1,
$$

has no solution in $(0, \pi)$ and has precisely one solution in $(n \pi,(n+1) \pi)$, $n=1,2, \ldots$, as can be seen from the graphs of $y=\tan x$ and $y=x$. Therefore,

$$
u=n \pi+\arctan (u / c), \quad c>1, n=1,2, \ldots,
$$


has a unique solution $u_{n}$ in $(n \pi,(n+1) \pi)$. The successive approximations

$$
u_{n, 1}=n \pi, \quad u_{n, k+1}=n \pi+\arctan \left(u_{n, k} / c\right), \quad k=1,2, \ldots,
$$

will converge to $u_{n}$. With this notation (1.6) is

$$
\sum_{n=1}^{\infty} \frac{1}{u_{n}^{2}}=\frac{1}{L^{2}}
$$

Now consider the function

$$
f(z):=\tan z-z / c
$$

It is clear that $0, \pm u_{n}$, and $\pm i L$ are zeros of $f(z)$ and its poles are $\pm\left(n-\frac{1}{2}\right) \pi$, $n=1,2, \ldots$. To see that $f$ has no other zeros note that $f(z)=0$ if and only if

$$
\frac{1}{c}=\frac{\tan z}{z}=\sum_{n=1}^{\infty} \frac{-2}{z^{2}-(n-1 / 2)^{2} \pi^{2}} .
$$

But the imaginary part of the above series does not vanish if $\operatorname{Im} z^{2} \neq 0$, hence, $f$ has no zeros other than $\pm u_{n}$ and $\pm i L$. Let

$$
C_{k}:=\text { a square with vertices } \pm k \pi \pm i k \pi, \quad k=1,2, \ldots
$$

It is easy to see that there is a constant $M$ independent of $k$ such that

$$
\left|f^{\prime}(z) / f(z)\right| \leq M \text { for all } z \in C_{k} \text { and all } k, k=1,2, \ldots
$$

[16]. Therefore,

$$
\int_{C_{k}} \frac{f^{\prime}(z)}{z^{2} f(z)} d z \rightarrow 0 \text { as } k \rightarrow \infty .
$$

Now the residue of $z^{-2} f^{\prime}(z) / f(z)$ at $\pm u_{n}$ is $1 /\left(u_{n}\right)^{2}$, while the residue of $z^{-2} f^{\prime}(z) / f(z)$ at $\pm i L$ is $-1 / L^{2}$. Furthermore the residues of $z^{-2} f^{\prime}(z) / f(z)$ at $z= \pm\left(n-\frac{1}{2}\right) \pi$ and at $z=0$ are equal to $-\left[\left(n-\frac{1}{2}\right) \pi\right]^{-2}$ and $2 c /[3(c-1)]$, respectively. Therefore,

$$
2 \sum_{n=1}^{\infty} \frac{1}{u_{n}^{2}}-\frac{2}{L^{2}}-2 \sum_{n=1}^{\infty} \frac{4}{(2 n-1)^{2} \pi^{2}}+\frac{2 c}{3(c-1)}=0
$$

Now the substitution of $\sum_{n \geq 1}(2 n-1)^{-2}=\pi^{2} / 8$ in the above equality establishes (1.6).

It is interesting to note that if we integrate $[z(z-u)]^{-1} f^{\prime}(z) / f(z)$ along $C_{k}$ and repeat the steps in the above proof we will discover a generalization of (1.6). To see this, define $g(z)$ by

$$
g(z):=\frac{1}{z(z-u)} \frac{f^{\prime}(z)}{f(z)}=\frac{c-\cos ^{2} z}{z(z-u)(c \sin z-z \cos z) \cos z}
$$


It is easy to see that

$$
\begin{aligned}
& \operatorname{Res}[g(z) ; z= \pm i L]=\frac{1}{ \pm i L( \pm i L-u)}, \\
& \operatorname{Res}\left[g(z) ; z= \pm u_{n}\right]=\frac{1}{ \pm u_{n}\left( \pm u_{n}-u\right)}, \\
& \operatorname{Res}[g(z) ; z=u]=\frac{c-\cos ^{2} u}{u(c \sin u-u \cos u) \cos u}, \\
& \operatorname{Res}[g(z) ; z=0]=-\frac{1}{u^{2}}, \\
& \operatorname{Res}\left[g(z) ; z= \pm\left(n-\frac{1}{2}\right) \pi\right]=\frac{-1}{ \pm(n-1 / 2) \pi[ \pm(n-1 / 2) \pi-u]},
\end{aligned}
$$

where

$$
\begin{gathered}
u_{n}:=n \pi+\arctan \frac{n \pi+\arctan \frac{n \pi+\arctan \cdots}{c}}{c} \\
L:=c \tanh (c \tanh (c \cdots)), \quad \text { and } c>1 .
\end{gathered}
$$

Furthermore,

$$
\int_{C_{k}} \frac{f^{\prime}(z)}{z(z-u) f(z)} d z \rightarrow 0 \quad \text { as } k \rightarrow \infty .
$$

Therefore, we proved

$\frac{c-\cos ^{2} x}{x(c \sin x-x \cos x) \cos x}=\frac{1}{x^{2}}+\frac{2}{L^{2}+x^{2}}+\sum_{n=1}^{\infty} \frac{2}{x^{2}-u_{n}^{2}}+\sum_{n=1}^{\infty} \frac{2}{(n-1 / 2)^{2} \pi^{2}-x^{2}}$.

We replaced $u$ by $x$ in the above formula. The last sum on the right-hand side is $(\tan x) / x$. Thus we have established the Mittag-Leffler expansion

$$
\frac{2}{L^{2}+x^{2}}+\sum_{n=1}^{\infty} \frac{2}{x^{2}-u_{n}^{2}}=\frac{1-(\sin (2 x) / 2 x)}{x^{2} \cos x[(\sin x) / x-(\cos x) / c]}-\frac{\tan x}{x} .
$$

Clearly (1.6) follows from (2.7) by letting $x \rightarrow 0$. It is worth noting that other identities involving power sums of $u$ also follow from (2.6) by expanding both sides of (2.6) in powers of $z$ and then equating coefficients of various powers of $x$.

We now discuss the functional equations (1.7) and

$$
f\left(z^{2}\right)=1-c z^{3}+c z^{3}\left(1-c z^{3}\right) f(1 / z) .
$$

Recall that $f$ of (1.8) satisfies (1.7) for all $x$ and all $c, c \neq 0,-1$. If $|c-1|<1$ and $f$ is right continuous at $x=0$, then $f(x):=[(1+x / c) /(c+1)]$ on $[0,1)$. To see this assume the contrary and let $f_{1}(x)$ and $f_{2}(x)$ be two solutions of (1.7) which are right continuous at $x=1$. Thus $h(x):=f_{1}(x)-f_{2}(x)$ satisfies

$$
h\left(x^{2}\right)=(x-c) h(x) \text {. }
$$

Clearly $h(1)=0$. Iterating (2.9) we get

$$
h(x)=(1-c)^{n} h\left(x^{2^{-n}}\right) \prod_{k=1}^{n}\left[1+\left(x^{2^{-k}}-1\right) /(1-c)\right] .
$$


When $n \rightarrow \infty$, since $|1-c|<1$, the above relationship implies $h(x) \equiv 0$ for $x$ in the complex plane cut along $(-\infty, 0)$. We believe that $f$ of $(1.8)$ is the only measurable solution to (1.7), $0 \leq x<\infty$, and it is likely that (1.7) will have additional nonmeasurable solutions. As far as (2.8) is concerned we proved that $f(z)=\left(1-c^{2} z^{3}\right) /\left(1+c^{3}\right)$ is the only solution to $(2.8)$ which is real analytic on a neighborhood of $z=1$. This also raises questions about how much local or global smoothness assumptions are necessary to guarantee that (2.8) will have a unique solution.

\section{Continued FRACTIONS}

Recall that the $q$ shifted factorial is

$$
\left(a ; q_{0}\right):=1, \quad(a ; q)_{n}=\prod_{k=1}^{n}\left(1-a q^{k-1}\right), \quad n=1,2, \ldots \text { or } n=\infty .
$$

For convenience when $n=\infty$ we need to assume $|q|<1$. We will use this notation in this section.

We now come to the continued fraction evaluation (1.3). Set

$$
X:=\frac{c \sinh x}{c \sinh x+\frac{1}{c \sinh (x+y)+\frac{1}{c \sinh (x+2 y)+\cdots}} .}
$$

It is clear that the evaluation of $X$ is equivalent to the evaluation of (1.3). Let $N_{n}(x, y)$ and $D_{n}(x, y)$ be the partial numerators and denominators of the above continued fraction, respectively. They satisfy the initial conditions

$$
\begin{array}{ll}
N_{0}(x, y)=0, & N_{1}(x, y)=c \sinh x, \\
D_{0}(x, y)=1, & D_{1}(x, y)=c \sinh x
\end{array}
$$

and the three-term recurrence relation

$$
f_{n+1}=c \sinh (x+n y) f_{n}+f_{n-1}, \quad n>0, f_{n}=N_{n}(x, y) \text { or } D_{n}(x, y) .
$$

We set

$$
q:=e^{2 y}, \quad u:=e^{2 x}
$$

hence,

$$
f_{n+1}=\frac{c}{2 \sqrt{u} q^{n / 2}}\left(u q^{n}-1\right) f_{n}+f_{n-1} .
$$

In order to identify the $N_{n}$ 's and $D_{n}$ 's in terms of the polynomials in [1] we need to renormalize the $N_{n}$ 's and $D_{n}$ 's (hence also the $f_{n}$ 's) via

$$
\begin{aligned}
& N_{n}(x, y)=i^{n} q^{n(2-n) / 4} \nu_{n}(x, y), \\
& D_{n}(x, y)=i^{n} q^{n(2-n) / 4} \delta_{n}(x, y), \\
& f_{n}=i^{n} q^{n(2-n) / 4} g_{n} .
\end{aligned}
$$

The recurrence relation $(3.5)$ is transformed to

$$
g_{n+1}=\frac{i c q^{-1 / 4}}{2 \sqrt{u}}\left(1-u q^{n}\right) g_{n}-q^{n-1} g_{n-1}, \quad n>0, g_{n}=\delta_{n}(x, y) \text { or } \nu_{n}(x, y)
$$


Al-Salam and Ismail [1] studied the polynomials $\left\{U_{n}(x ; a, b)\right\}$ generated by

$$
\begin{aligned}
U_{0}(x ; a, b):= & 1, \quad U_{1}(x ; a, b):=x(1+a), \\
U_{n+1}(x ; a, b)= & x\left(1+a q^{n}\right) U_{n}(x ; a, b) \\
& -b q^{n-1} U_{n-1}(x ; a, b), \quad n>0 .
\end{aligned}
$$

It is easy to see that by renormalizing $x$ and $U_{n}$, there is no loss of generality in taking $b=1$. The parameter $b$ was kept, however, for convenience. Clearly $\delta_{n}(x, y)=U_{n}\left(i c q^{-1 / 4} u^{-1 / 2} / 2 ;-u, 1\right)$; hence,

$$
\begin{aligned}
D_{n}(x, y) & =i^{n} q^{n(2-n) / 4} U_{n}\left(\frac{i c}{2} u^{-1 / 2} q^{-1 / 4} ;-u, 1\right) \\
& =i^{n} q^{n(2-n) / 4} U_{n}\left(\frac{i c}{2} e^{-x-y / 2} ;-e^{2 x}, 1\right) .
\end{aligned}
$$

Using the representation $[1$, p. 274$]$

$$
U_{n}(x ; a, b)=\sum_{k=0}^{[n / 2]} \frac{(-a ; q)_{n-k}(q ; q)_{n-k} x^{n-2 k}(-b)^{k}}{(-a ; q)_{k}(q ; q)_{k}(q ; q)_{n-2 k}} q^{k(k-1)},
$$

we get

$$
D_{n}(x, y)=\sum_{k=0}^{[n / 2]} \frac{\prod_{j=0}^{n-k-1}[c \sinh (x+j y) / \sinh ((j+n-k+1) y)]}{\prod_{j=0}^{k-1} c \sinh (x+j y) \sinh ((j+1) y)} .
$$

On the other hand it is clear from (3.1) and (3.2) that

$$
N_{n}(x, y)=c \sinh x D_{n-1}(x+y, y),
$$

which together with (3.10) gives an explicit representation for $N_{n}(x, y)$. Since the continued fraction $X$ is invariant under $x \rightarrow-x, y \rightarrow-y, c \rightarrow-c$, we may restrict ourselves to the case

$$
\operatorname{Re} y<0, \quad \text { that is, }|q|<1 .
$$

From the asymptotic formula

$$
U_{n}(x ; a, b) \approx x^{n}(-a ; q)_{\infty} \sum_{k=0}^{\infty} \frac{(-b)^{k}}{(q ; q)_{k}(-a ; q)_{k}} x^{-2 k} q^{k(k-1)}, \quad|q|<1,
$$

which implies

$$
\begin{aligned}
D_{n}(x, y) \approx & \left(-\frac{c}{2}\right)^{n} \exp \left\{-n x-n(n-1) \frac{y}{2}\right\}\left(e^{2 x} ; e^{2 y}\right)_{\infty} \\
& \times \sum_{k=0}^{\infty} \frac{\exp [k(x+k y)]}{\prod_{j=1}^{k}\{c \sinh k y c \sinh [x+(j-1) y]\}},
\end{aligned}
$$

holding for $\operatorname{Re} y<0$. Now (1.3) follows from (3.11), (3.12), and the fact that $N_{n}(x, y) / D_{n}(x, y) \rightarrow X$ as $n \rightarrow \infty$.

Note that Gosper did not state conditions under which (1.3) holds. Obviously it holds when $\operatorname{Re} y>0$, and if $\operatorname{Re} y<0$ we must perform the change of variables $(x, y, c) \rightarrow(-x,-y,-c)$, which does not change the continued fraction but gives the correct right member of (1.3). 
Ismail and Mulla [12] essentially proved the continued fraction representation

$$
\frac{2}{2 x-a-\frac{1}{2 x-a q-\frac{1}{2 x-a q^{2}-\cdots}}}=2 B F_{a q}(x) / F_{a}(x),
$$

where

$$
F_{a}(x):=\sum_{k=0}^{\infty} \frac{(-a B)^{k} q^{k(k-1) / 2}}{\left(B^{2} ; q\right)_{k+1}(q ; q)_{k}}
$$

and $A, B=x \pm\left(x^{2}-1\right)^{1 / 2},|B| \leq|A|$. The relationship (3.13) holds if $|B|<|A|$. Before we proceed with the choices

$$
a=-c, q=z, B=-y, \text { so } A=-1 / y \text {, and }|y| \leq 1 \text {, }
$$

we want to establish an alternate representation for $F_{a}(x)$. First use the fact

$$
\left(B^{2} ; q\right)_{k+1}=\left(B^{2} ; q\right)_{\infty} /\left(B^{2} q^{k+1} ; q\right)_{\infty} ;
$$

then apply Euler's identity [3]

$$
(s ; q)_{\infty}=\sum_{j=0}^{\infty} \frac{(-s)^{k} q^{k(k-1) / 2}}{(q ; q)_{k}}
$$

to get

$$
F_{a}(x)=\frac{1}{\left(B^{2} ; q\right)_{\infty}} \sum_{k=0}^{\infty} \frac{(-a B)^{k} q^{k(k-1) / 2}}{(q ; q)_{k}} \sum_{j=0}^{\infty} \frac{\left(-B^{2}\right)^{j}}{(q ; q)_{j}} q^{j(j-1) / 2} q^{j(k+1)} .
$$

Interchanging the $k$ and $j$ sums, summing the $k$ sum by Euler's theorem we arrive at the representation

$$
F_{a}(x)=\frac{(a B ; q)_{\infty}}{\left(B^{2} ; q\right)_{\infty}} \sum_{j=0}^{\infty} \frac{\left(-B^{2}\right)^{j} q^{j(j+1) / 2}}{(q ; q)_{j}(a B ; q)_{j}} .
$$

It is now straightforward to obtain (1.4) from (3.13), (3.14), and (3.16).

It is important to note that (1.4) holds if $|y|<1$, but if $|y|>1$ we must interchange $y$ and $1 / y$.

Finally we come to (1.5). It is easier to prove (1.5) directly rather than to try to get it from known results $[12,13]$. Let $N_{n}$ and $D_{n}$ be the partial numerators and denominators of the reciprocal of the continued fraction in (1.5). Thus

$$
\begin{gathered}
N_{0}=0, \quad N_{1}=1, \quad D_{0}=1, \quad D_{1}=c, \\
w_{n+1}=c z^{n} w_{n}+w_{n-1}, \quad n>0, \quad w_{n}=D_{n} \text { or } N_{n} .
\end{gathered}
$$

Let $W(t)=\sum_{n \geq 0} t^{n} D_{n}$ to see that (3.17) for $w_{n}=D_{n}$ is equivalent to the functional equation

$$
\left(1-t^{2}\right) W(t)=1+t c W(z t) .
$$

Thus when $|z|<1$ we iterate the above functional equation and use $W\left(z^{n} t\right) \rightarrow$ 1 as $n \rightarrow \infty$ to get

$$
W(t)=\sum_{k=0}^{\infty} \frac{(c t)^{k} z^{k(k-1) / 2}}{\left(t^{2} ; z^{2}\right)_{k+1}}
$$


and now Darboux's method [14] yields

$$
D_{n} \approx \frac{1}{2} \sum_{k=0}^{\infty} \frac{c^{k} z^{k(k-1) / 2}}{\left(z^{2} ; z^{2}\right)_{k}}\left[1+(-1)^{n+k}\right]
$$

Thus

$$
D_{2 n}=\sum_{k=0}^{\infty} \frac{c^{2 k} z^{k(2 k-1)}}{\left(z^{2} ; z^{2}\right)_{2 k}} \quad \text { and } \quad D_{2 n+1} \approx \frac{c}{1-z^{2}} \sum_{k=0}^{\infty} \frac{c^{2 k} z^{k(2 k+1)}}{\left(z^{4} ; z^{2}\right)_{2 k}} .
$$

From (3.17) it is clear that $N_{n}$ is $D_{n-1}$ with $c$ replaced by $c z$. Therefore, (3.18) gives

$$
\begin{aligned}
& \lim _{n \rightarrow \infty} \frac{N_{2 n}}{D_{2 n}}=\left[\sum_{k=0}^{\infty} \frac{c^{2 k+1} z^{(k+1)(2 k+1)}}{\left(z^{2} ; z^{2}\right)_{2 k+1}}\right] /\left[\sum_{k=0}^{\infty} \frac{c^{2 k} z^{k(2 k-1)}}{\left(z^{2} ; z^{2}\right)_{2 k}}\right], \\
& \lim _{n \rightarrow \infty} \frac{N_{2 n+1}}{D_{2 n+1}}=\left[\sum_{k=0}^{\infty} \frac{c^{2 k} z^{k(2 k+1)}}{\left(z^{2} ; z^{2}\right)_{2 k}}\right] /\left[\sum_{k=0}^{\infty} \frac{c^{2 k+1} z^{k(2 k+1)}}{\left(z^{2} ; z^{2}\right)_{2 k+1}}\right] .
\end{aligned}
$$

Thus when $|z|<1$ the convergents (approximants) of even and odd orders will converge to different limits, and the continued fraction (1.5) will diverge.

We now come to the case $|z|>1$. Let $w_{n}=z^{n(n-1) / 2} v_{n}$ so that

$$
v_{n+1}=c v_{n}+z^{1-2 n} v_{n-1}, \quad n>0
$$

Comparing (3.7b) and (3.19) we see that the reciprocal of the continued fraction in (1.5) equals

$$
X:=\frac{1}{c+\frac{z^{-1}}{c+\frac{z^{-3}}{c+\cdots}}}
$$

But $X$ is the continued fraction associated with the $U_{n}$ 's generated by (3.7a) and (3.7b) when $a=0, x=c, b=-z$, and $q=z^{-2}$. From (5.3) in [1] we obtain

$$
X=\frac{1}{c}\left[\sum_{n=0}^{\infty} \frac{c^{-2 n}}{\left(z^{-2} ; z^{-2}\right)_{n}} z^{-n(2 n+1)}\right] /\left[\sum_{n=0}^{\infty} \frac{c^{-2 n}}{\left(z^{-2} ; z^{-2}\right)_{n}} z^{-n(2 n-1)}\right]
$$

This shows that the left side of (1.5) is the reciprocal of the right side in (3.21). Thus we proved that for $|z|>1$ we have

$$
\begin{aligned}
c+ & \frac{1}{c z}+\frac{1}{c z^{2}+\cdots} \\
& =c\left[\sum_{n=0}^{\infty} \frac{c^{-2 n}}{\left(z^{-2} ; z^{-2}\right)_{n}} z^{-n(2 n-1)}\right] /\left[\sum_{n=0}^{\infty} \frac{c^{-2 n}}{\left(z^{-2} ; z^{-2}\right)_{n}} z^{-n(2 n+1)}\right] .
\end{aligned}
$$

Gosper [9, p. 7] indicated that both sides of (1.5) equal

$$
\begin{aligned}
c+ & \frac{1}{c z}-\frac{1}{c^{3} z^{4}}+\frac{1}{c^{5} z^{7}}+\frac{1}{c^{5} z^{9}}-\frac{1}{c^{7} z^{10}}-\frac{2}{c^{7} z^{12}}+\frac{1}{c^{9} z^{13}}-\frac{1}{c^{7} z^{14}} \\
+ & \frac{3}{c^{9} z^{15}}-\frac{1+c^{4}}{c^{11} z^{16}}+\frac{3}{c^{9} z^{17}}-\frac{4}{c^{11} z^{18}}+O\left(z^{-19}\right) .
\end{aligned}
$$


It is easy to verify that both sides of $\left(1.5^{\prime}\right)$ have the above asymptotic series as $z \rightarrow \infty$.

\section{MORE ON ITERATIONS}

We now come to (1.10)-(1.13). We will give a proof of (1.10) and only outline proofs of (1.11)-(1.13) because the proofs of the four are similar beyond the identification of the iteration operator.

Proof of (1.10). Let us denote the left-hand side of (1.10) by $f(a, b, c)$. By subtracting the term $n=1$ from $f(a, b, c)$ and then replacing the summation index $n$ by $n+1$ in the remaining series we discover

$$
\begin{aligned}
f(a, b, c)= & 2 \cos (b-a / 2) \sin (3 b+c+a / 2) \\
& -2 \cos (b-a / 2) f(-a / 2, b, c+3 b) .
\end{aligned}
$$

If we denote the left side of $(1.10)$ by $g(a, b, c)$ then after using some elementary trigonometric identities we find that $g(a, b, c)$ also satisfies (4.1). The iteration operator $S$ acts on $a$ and $c$ by $S(a, c)=(-a / 2, c+3 b)$ and $b$ remains invariant. Iterate (4.1), with $f$ replaced by $g$. Now (1.10) follows from the boundedness of $g(a, b, c)$ as a function of $a$ and $c$ when $b \neq 0,-1, \ldots$ and the assumption $|\cos b|<\frac{1}{2}$.

Proof of (1.11). The iteration operator is $S z=1 / \sqrt{z}$. Iterate the functional equation

$$
f(z)+\frac{1}{2} f\left(\frac{1}{\sqrt{z}}\right)=-\frac{a+b / \sqrt{z}}{2(1+1 / \sqrt{z})},
$$

which is satisfied by both sides of (1.11).

Proof of (1.12). Both sides of (1.12) satisfy the functional equation

$$
\begin{aligned}
f(z)-f(1 / \sqrt{z}) & =-\left(a z^{-1 / 6}+b z^{-5 / 6}\right)\left(z^{1 / 2}-z^{-1 / 2}\right) \\
& =\left(a z^{-2 / 3}+b z^{-4 / 3}\right)(1-z) .
\end{aligned}
$$

Apply the iteration operator $S: z \rightarrow 1 / \sqrt{z}$.

Proof of (1.13). The functional iteration here is $(a, b, c) \rightarrow(2 a, b, c-b)$. Both sides of (1.13) satisfy the functional equation

$$
f(a, b, c)-\frac{1}{2 \cosh (a+b)} f(2 a, b, c-b)=\frac{\sinh (2 a-b+c)}{2 \cosh (a+b)} .
$$

The rest is straightforward.

It is interesting to note that the term $[\sinh (a+b) \sinh (c-2 b)] /(\sinh b)$ on the right-hand side of (1.13) is a particular solution of the functional (4.4), while the other term on the right-hand side of (1.13) is a solution of the corresponding homogeneous functional equation.

Another identity of Gosper is

$$
\sum_{n=0}^{\infty} 2^{n} \sin \left(c+n b+\frac{a}{2^{n}}\right) \prod_{k=1}^{n} \cos \left(b+\frac{a}{2^{n}}\right)=\frac{\sin (a+b) \sin (2 b-c)}{\sin b}
$$

We leave the proof of $(4.5)$ to the interested reader as an exercise in the application of the iteration principle. 
Gosper [7] used matrices of the form $\left[\begin{array}{ll}* & * \\ 0 & 1\end{array}\right]$ to prove (1.9), where ${ }^{*}$ stands for suitably chosen elements. Our method of iteration uses two-term recursions. Thus Gosper's matrix methods in the case of $n=2$ is somehow related, and possibly equivalent, to the use of two-term recursions. It is likely that his higher method for higher-order matrices correspond to the use of recursion relations of higher order. This point is still under investigation.

\section{ACKNOWLEDGMENT}

We gratefully acknowledge Bill Gosper's interest and input into this work. His numerous electronic mail messages containing challenges and suggestions were the inspiration that shaped this work. The first named author also acknowledges the hospitality of the National University of Colombia in Bogota where part of this research was done.

\section{REFERENCES}

1. W. Al-Salam and M. E. H. Ismail, Orthogonal polynomials associated with the RogersRamanujan continued fraction, Pacific J. Math. 104 (1983), 269-283.

2. R. Askey and M. E. H. Ismail, Recurrence relations, continued fractions and orthogonal polynomials, Mem. Amer. Math. Soc., vol. 49, no. 300, Amer. Math. Soc., Providence, RI, 1984.

3. G. Gasper and M. Rahman, Basic hypergeometric series, Cambridge Univ. Press, Cambridge, 1990.

4. __ An infinite bibasic summation formula and some quadratic transformation formulas, Canad. J. Math. 42 (1990), 1-27.

5. I. Gessel and D. Stanton, Strange evaluations of hypergeometric series, SIAM J. Math. Anal. 13 (1982), 185-308.

6. $\longrightarrow$, Applications of q-Lagrange inversion to basic hypergeometric series, Trans. Amer. Math. Soc. 277 (1983), 173-201.

7. R. W. Gosper, Strip mining in the abandoned ore fields of nineteenth century mathematics, Computers in Mathematics (D. V. Chudnovsky and R. D. Jenks, eds.), Lecture Notes in Pure and Appl. Math., vol. 125, Marcel Dekker, New York, 1990, pp. 261-284.

8. __ Some identities for your amusement, Ramanujan Revisited (G. Andrews, R. Askey, B. Berndt, K. Ramanathan, and R. Rankin, eds.), Academic Press, New York, 1988, pp. 607-609.

9. __ Material from Bill Gosper's computers \& mathematics talk, M. I. T., June 1989.

10. _ private communication, July 11, 1991.

11. R. W. Gosper, M. E. H. Ismail, and R. Zhang, On some strange summation formulas, Illinois J. Math. 37 (1993), 240-277.

12. M. E. H. Ismail and F. S. Mulla, On the generalized Chebyshev polynomials, SIAM J. Math. Anal. 18 (1987), 243-258.

13. W. Jones and W. Thron, Continued fractions, analytic theory and applications, Cambridge Univ. Press, Cambridge, 1984.

14. F. W. J. Olver, Asymptotics and special functions, Academic Press, New York, 1974.

15. L. J. Slater, Hypergeometric series, Cambridge Univ. Press, Cambridge, 1966.

16. E. T. Whittaker and G. N. Watson, A course of modern analysis, fourth ed., Cambridge Univ. Press, Cambridge, 1966.

Department of Mathematics, University of South Florida, Tampa, Florida 33620

Department of Mathematics, National University of Colombia, Bogota, Colombia

Department of Mathematics, University of South Florida, Tampa, Florida 33620 